\section{Odd Vibration Sources}

Tina (Weatherby) Carvalho, University of Hawaii at Manoa tina@pbrc.hawaii.edu

Chasing sources of vibration seems to be a never-ending problem in microscopy. Here are the approaches we've taken to two different sources of vibration affecting our Hitachi S-800 FESEM. One of the first problems was with a window air conditioning unit in the room that was needed to augment our so-

\section{A Fool-Proof Method For Mounting Serial Sections} On Single Hole Grids

Continued from 27

with stain precipitation if you use very clean water and transfer the grid through a sufficient number of water droplets $(6-12$ recommended).

6) The final step is to transfer the grid to a film suspended over the hole in a Plexiglas slide and let it dry down. The sections will now be stuck to the film with NO wrinkles and minimum breakage. When ready to view, just punch out around the grid with the tip of your forceps, grab the grid and insert into the microscope.

Believe me....the sections will still be there at the end!

I found that as long as the sections cover a substantial portion of the open area of the grid, carbon coating was not essential. I used to do 50-100 grids worth of serial sections without losing any. The films on the plastic slides would hold for months so I could make a lot and store until needed. The method really works...do give it a try.

\section{Remove the Veil from SEM Samples!} JACRON'T

\section{in-sifu Plasma Cleaning stops confamination inside your SEM!}

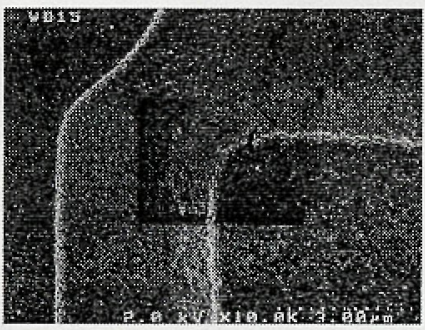

Unwanted Artifact

A short SEM scan started a contamination deposit.

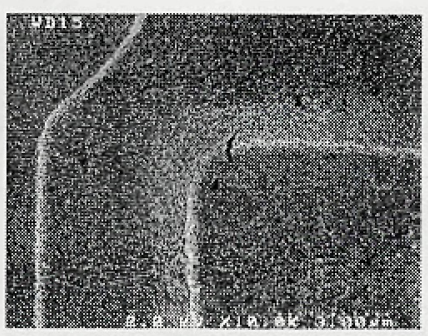

After Evactron Cleaning 2 minutes of cleaning in-situ removed the deposit and prevented new artifacts.

\section{EI scientific}

SEM-CLEANTM Systems for SEMs

3124 Wessex Way, Redwood City, CA 94061

(650) 369-0133, FAX (650) 363-1659

Email: RVaneXEI@concentric.net

www semclean.com called central system. The existing window unit vibrated the window, walls, and floor. One solution may have been to buy a split unit so that the compressor was outdoors, but there was a moratorium on buying new air conditioning units. An additional problem is that the split units do not dehumidify well enough for the tropics. Our physical plant people came up with a design that worked with the existing unit. The air conditioner was mounted on a welded steel frame set into concrete in the ground outside the window. Where it would have been mounted in the window in a wooden frame, the wood was instead replaced with a heavy rubber bellows. For magnifications of up to about $80,000 \times$ we can leave the air conditioner running and we do not see any changes as the compressor cycles on and off, but we still turn off the unit at greater magnifications. Our only problem now is that people often use the steel frame outside as a bicycle rack, resulting in more activity under that window and against the wall than we would prefer!

A second source of vibration turned out to be the metal shrouding over the ion getter pumps mounted behind the column. Although the column has its own, isolated suspension, we still noticed jaggies on the screen at high magnifications when people spoke in the room. Further investigation (well, OK, it was an accident) proved that when the metal shrouding around the pump was removed that particular source of vibration was eliminated. It turns out that the pump shrouding was acting like a drum and transmitting audio noise to the column. Interestingly, it did not seem to transmit the noise of the rotary pumps. I simply leave the shrouding off these days, and Hitachi has subsequently redesigned the shrouding to be perforated to minimize the drum effect.

\begin{tabular}{ccc|}
\hline Your Source For All \\
Polaroid Products
\end{tabular}

